\title{
ИЗУЧЕНИЕ МОЛЕКУЛЯРНО-ГЕНЕТИЧЕСКИХ АНОМАЛИЙ В ОПУХОЛЯХ ГИПОФИЗА. СОВРЕМЕННОЕ СОСТОЯНИЕ ПРОБЛЕМЫ
}

\author{
Лапшина A.M. \\ ФГБУ «НМИЦ эндокринологии» Минздрава России, Москва.
}

Последние годы проводится интенсивное изучение опухолей гипофиза при помощи различных методов молекулярно-генетического (экзомного и геномного) анализа с целью определения генов кандидатов, биомаркеров и молекулярных механизмов.

МАТЕРИАЛЫ И МЕТОДЫ: актуальнЫм является вопрос использования видов образцов биологического материала (кровь или ткань), ввиду трудной доступности ткани опухоли гипофиза для указанных исследований на дооперационном этапе. Изучается роль драйверных мутаций (GNAS и USP-8) в регуляции сигнальных путей и клеточного цикла, изменении транскриптомного профиля в опухолях гипофиза, эпигенетических факторов и микроокружения. На сегодняшний день наиболее важным является вопрос в отношении экстраполяции данных, полученных в результате фундаментальных исследований, на такие клинические проблемы, как определение прогноза и чувствительности опухолей гипофиза к различным видам терапии и разработки новых видов лечения. В частности для определения положительного эффекта от различных видов терапии требуется углубленное понимание молекулярных механизмов таких процессов, как пролиферативная активность, гормональная секреция, инвазивный рост. В настоящее время наиболее широко изучены опухоли гипофиза, развившиеся в рамках наследственных синдромов (5\%), в отношении мутаций из зародышевых клеток (AIP, MEN1 и др.). Однако остальная, большая часть спорадических опухолей (около 95\%) представлена мутациями во множественных генах, несмотря на то, что опухоли имеют одну локализацию.

Выводы: таким образом, подробное изучение зародышевых и соматических геномных альтераций, нарушение регуляции сигнальных путей, факторов микроокружения в опухолях гипофиза позволит выявить механизмы для воздействия новых видов терапии и диагностики.

КЛЮЧЕВЫЕ СЛОВА: сигнальные пути, клеточный чикл, транскриптомный профиль в опухолях гипофиза. 